DOI 10.32370/2018_11_4

\title{
Computer Crime
}

\author{
Dr. Umar Lawal Aliyu \\ Faculty of Management, Department of Business Administration \\ LIGS University Hawaii, USA \\ lawalacademy@gmail.com
}

\begin{abstract}
Crime is an action or omission, which constitutes an offence and is punishable by law. A crime is an offence that merits community condemnation and punishment, usually by way of fine or imprisonment. Crime takes place when a person deliberately practices deception in order to gain something unlawfully or unfairly. While crime is most commonly committed to obtain benefits of value, it sometimes occurs solely for deceiving another person or entity. Computer crime alternatively referred to as cybercrime, e-crime, electronic crime, or hi-tech crime is an act performed by a knowledgeable computer user, sometimes referred to as a hacker that illegally browses or steals a company's or individuals private information. In some cases, this person or group of individuals may be malicious and destroy or otherwise corrupt the computer or data files. Cybercrime may threaten a person or a nation's security and financial health. Issues surrounding these types of crimes have become high profile, particularly those surrounding hacking, copyright infringement, unwarranted mass-surveillance, extortion, child pornography, and child grooming. This paper focused on the causes, types, detection and prevention of computer crime. The paper also reviews the various forms and types of computer crime practice, their impact and recommendations that will curtail this bad menace.
\end{abstract}

Keywords: Crime, Computer crime, Data, Detection, Illegal, Offence, Prevention, Punishment

\subsection{INTRODUCTION}

The U.S. Department of Justice (DOJ), in its manual on computer crime, defines such crime as "any violations of criminal law that involve knowledge of computer technology for their perpetration, investigation, or prosecution." According to Professor Babatope Longe of Computer Security and Dean, Caleb Business School, Caleb University, Imota, Lagos State, cyber criminality and cyber victimization are crimes tied to the exploration of human frailties such as greed, gullibility and the untamed quest for getting rich syndrome and not crimes that are necessarily influenced by social factors such as poverty, inequality and unemployment.

Cybercrime, or computer-oriented crime, is the crime that involves a computer and a network. The computer may have been used in the commission of a crime, or it may be the target. Cybercrimes can be defined as: "Offences that are committed against individuals or groups of individuals with a criminal motive to intentionally harm the reputation of the victim or cause physical or mental harm, or loss, to the victim directly or indirectly, using modern telecommunication networks such as Internet (networks including but not limited to Chat rooms, emails, notice boards and groups) and mobile phones (Bluetooth/SMS/MMS)". Vices such as hacking, phishing, cyber stalking, online pornography, advance fee fraud and cyber 
terrorism are examples of those cybercrimes and are traceable all over the world. Cybercrime includes any type of illegal scheme that uses one or more components of the Internet (chat rooms, email, message boards, websites, and auctions) to conduct fraudulent transactions or transmit the proceeds of fraud to financial institutions or to others connected with the scheme. Cybercrime also applies to generating spam emails, downloading viruses or spyware to computer, harassing another through the Internet, child pornography, and solicitation of prostitution online. Perhaps the most prominent form of cybercrime is identity theft, in which criminals use the Internet to steal personal information from other users.

The thrust of the research thesis is to examine computer crime, types of computer crime and give recommendations in order to reduce the pitfall individuals, banks went through in the hands of cybercriminals in recent years.

\subsection{LITERATURE REVIEW}

\subsection{Theoretical Framework}

Throughout the past, several decades there have been numerous advances in electronic resources. Technologies such as cellular phones, pagers, home computers, the Internet, websites, and palm pilots have added another dimension to crime. While each of the networks that make up the Internet is owned by a public or private organization, no single organization or government owns or controls the Internet. Originally created to further defence, scientific and academic endeavours, the Internet which also affords users the ability to communicate via electronic mail ("e-mail"), grew slowly but steadily until 1994. At that time, the World Wide Web ("the Web"), the graphical user interface to the Internet, was introduced which prompted extraordinary growth in both the size and the use of the Internet.

Internet and the Web importance rocketed in the economy and globally with Ecommerce, Online businesses, credit card purchases over the Internet 24-hours-a-day and host of plenty goodies but also faced with cybercrime; generating spam emails, downloading viruses or

spyware to computer, harassing another through the Internet, child pornography, and solicitation of prostitution online. Cyber criminals use internet and computer technology to hack user's personal computers, smartphone data, and personal details from social media, business secrets, national secrets etc. Criminals who perform these illegal activities through the internet are called - Hackers. Though law enforcement agencies are trying to tackle this problem, it is growing regularly and many people have become victims of identity theft, hacking and malicious software.

\subsection{Causes of Computer Crime}

We are living in the modern era based on the technology. Our daily life depends on it, live with it. Therefore, nowadays the internet is a common name known to everyone. Millions of people globally on daily basis use computers and the internet. They are used in houses, 
schools, work and in fact have become part of our life and daily activities. Computers have made our life easier, it has brought so many benefits to the society but it has also brought some problems and cybercrimes is one of them. The Internet contains everything we need. Thus, people are using, depending on it more, and more.

However, causes of cybercrime can be attributed to the following below:

a. Negligence: Negligence provides a cyber-criminal the access and control over the computer system.

b. Greed: 'Greed' is one of the main causes for people falling prey to cybercrimes despite extensive exposure of its dangers by authorities and cautionary tales from cheated victims.

c. Loss of evidence: This has become a very common and obvious problem, which paralyzes the system behind the investigation of cyber-crime.

d. Complex: The computers run on operating systems and these operating systems are programmed of millions of codes. The human mind is imperfect, so they can do mistakes at any stage. The cyber criminals take advantage of these gaps.

e. Easy to access: Hackers can steal access codes, retina images, advanced voice recorders etc. that can fool biometric systems easily and bypass firewalls can be utilized to get past many security systems.

f. Capacity to store data in comparatively small space: This makes it a lot easier for the people to steal data from any other storage and use it for own profit.

\subsection{Types of Cyber Crime}

a. Child pornography and Abuse: Making or distributing child pornography.

b. Hacking: Refers to unauthorized intrusion into a computer or a network. Hacker accesses personal or sensitive information without authorisation.

c. Piracy or Theft: This crime occurs when a person violates copyrights and downloads music, movies, games, and software. Copyright violation is stealing or using another person's Copyrighted material without permission.

d. Cracking: Breaking or deciphering codes that are being used to protect data.

e. Cyber terrorism: Hacking, threats, blackmailing towards a business or person.

f. Cyber bully and Cyber stalking: This is a kind of online harassment wherein the victim is subjected to a barrage of online messages and emails.

g. Identity theft: Pretending to be someone you are not.

h. Cybersquatting: Setting up a domain of another person or company with the sole intentions of selling it to them later at a premium price.

i. Creating Malware: Writing, creating, or distributing malware (e.g., viruses and spyware.)

j. Denial of Service attack: Overloading a system with so many requests it cannot serve normal requests.

k. Espionage: Spying on a person or business. 
1. Fraud - Manipulating data, e.g., changing banking records to transfer money to an account or participating in credit card fraud.

m. Harvesting: Collect account or other account related information on other people.

n. Human trafficking: Participating in the illegal act of buying or selling other humans.

o. Identity theft: Pretending to be someone you are not.

p. Illegal sales: Buying or selling illicit goods online including drugs, guns, and psychotropic substances.

q. Intellectual property theft: Stealing practical or conceptual information developed by another person or company.

r. IPR violation: An intellectual property rights violation is any infringement of another's Copyright, patent, or trademark.

s. Phishing: Deceiving individuals to gain private or personal information about that person.

t. Salami slicing: Stealing tiny amounts of money from each transaction.

u. Scam: Tricking people into believing something that is not true.

v. Slander: Posting libel or slander against another person or company.

w. Software piracy: Copying, distributing, or using software that is Copyrighted that you did not purchase.

x. Spamming: Distributed unsolicited e-mail to dozens or hundreds of different addresses.

y. Spoofing: Deceiving a system into thinking you are someone you really are not.

z. Typosquatting - Setting up a domain that is a misspelling of another domain.

aa. Unauthorized access - Gaining access to systems you have no permission to access.

bb. Wiretapping - Connecting a device to a phone line to listen to conversations.

\subsection{CONCLUSION}

Even though, computers are introduced with the view of making things easier for those who use them, it has become an avenue for the easiest fraud to take place in the whole world. It has one Logo: "Garbage in - Garbage Out. To tackle cybercrime effectively, establish multidimensional public-private collaborations between law enforcement agencies, the information technology industry, information security organizations, internet companies and financial institutions.

Also, use Strong Passwords, Be social media savvy, Secure your Mobile Devices, Protect your data, Protect your identity online, Keep your computer current with the latest patches and updates, Protect your computer with security software and always call the right person for help.

\section{References}

1. Causes of CyberCrime and Preventive Measures by Ravi Bandakkanavar | August 30, 2017

2. Causes of CyberCrime and Preventive Measures by Ravi Bandakkanavar 2017

3. Coding Freedom: The Ethics and Aesthetics of Hacking by E. Gabriella Coleman 2012 
4. Computer Hope Free Computer Help by Computer Hope since 2018

5. Cybercrime From Wikipedia, the free encyclopaedia

6. FTC Targets Computer Support Scams by Information Management, Vol. 47, No. 1, 2013

7. Greed, Cause of Cybercrime by Dayo Adesulu June 2015

8. Legal Services Commission of South Australia; Criminal and Traffic Offences - Revised 2017

9. New Global Cybercrime Calls for High Tech Cyber-Cops by McMahon, Richard; Bressler, Martin S.; Bressler, Linda 2016

10. Phishing, Pharming and Identity Theft by Brody, Richard G.; Mulig, Elizabeth; Kimball, Valerie 2009

11. Risk Factors in Computer-Crime Victimization by Kyung-Shick Choi 2010

12. USLegal; Cybercrimes Law and Legal Definition 